\title{
Eficacia reducida de derquantel y abamectina en ovinos y caprinos con Haemonchus sp resistentes a lactonas macrocíclicas
}

\author{
Cerutti, J.; Cooper, L..; Torrents, J.'; Suárez, G. ${ }^{2}$; Anziani, O.S.,2 \\ ${ }^{1}$ Facultad Ciencias Agropecuarias, Univ. Católica de Córdoba, Armada Argentina 3555, Córdoba, Argentina. \\ ${ }^{2}$ EEA INTA Rafaela, Santa Fe. E-mail: anziani.oscar@inta.gob.ar
}

\begin{abstract}
Resumen
Cerutti, J.; Cooper, L.; Torrents, J.; Suárez, G.; Anziani, O.S.: Eficacia reducida de derquantel y abamectina en ovinos y caprinos con Haemonchus sp resistentes a lactonas macrocíclicas. Rev. vet. 29: 1, 22-25, 2018. Se presentan las observaciones efectuadas sobre la actividad de una combinación antihelmíntica conteniendo un nueva droga, el derquantel (spiroindoles) adicionada con abamectina (lactonas macrocíclicas) en ovinos y caprinos naturalmente parasitados por Haemonchus $s p$. En el conteo de huevos, la eficacia de la combinación mostró porcentajes de reducción variables y dependientes de los estadios parasitarios intervinientes al momento del tratamiento y al status previo de resistencia a las lactonas macrocíclicas. Se observó una eficacia alta (mayor al 99\%) frente a la oviposición de estadios maduros de Haemonchus sp o cuando los antecedentes de las poblaciones parasitarias indicaban resistencia no marcada a las lactonas macrocíclicas. Por el contrario, eficacias inferiores ( 76 al 83\%) se observaron cuando las poblaciones de este parásito mostraban resistencia severa a las lactonas macrocíclicas y los estadios inmaduros constituían la mayor parte de la población parasitaria al momento del tratamiento. Las combinaciones antihelmínticas tienen un interesante potencial de uso frente a poblaciones parasitarias de Haemonchus $s p$ resistentes a los antihelmínticos, pero las presentes observaciones enfatizan la necesidad de evaluar a nivel de cada establecimiento la actividad de todos los antiparasitarios, sean estos formulados con viejas o nuevas drogas, si se pretende instaurar programas racionales para el control de estos nematodes.
\end{abstract}

Palabras clave: corderos, Haemonchus sp, derquantel, abamectina, eficacia.

\begin{abstract}
Cerutti, J.; Cooper, L.; Torrents, J.; Suárez, G.; Anziani, O.S.: Reduced efficacy of derquantel and abamectin in sheep and goats with Haemonchus sp resistant to macrocyclic lactones. Rev. vet. 29: 1, 22-25, 2018. A field study was carried out to determine the activity of an anthelmintic combination of derquantel (spiroindoles) plus abamectin (macrocyclic lactones) against Haemonchus $s p$ in sheeps and goats. The clinical efficacy of the combination showed variable egg count reduction percentage dependent on the parasitic stages involved at the time of treatment and the previous status of resistance to the macrocyclic lactones. High efficacy (greater than 99\%) was observed against oviposition of mature Haemonchus sp stages or when background of parasitic populations indicated moderate resistance to macrocyclic lactones. In contrast, lower efficiencies (76-83\%) were observed when populations of this parasite showed severe resistance to macrocyclic lactones and immature stages constituted the majority of the parasitic population at time of treatment. Anthelmintic combinations could have an interesting potential for prevent o manage the nematode resistance in small ruminants, but the present observations emphasize the need to evaluate at the level of each establishment the activity of all anthelmintic drenches formulated with new or old drugs.
\end{abstract}

Key words: lambs, Haemonchus sp, derquantel, abamectin, efficacy.

\section{INTRODUCCIÓN}

Los nematodes gastrointestinales constituyen el principal problema sanitario en los pequeños rumiantes. En tal contexto, el género Haemonchus es conside-

Recibido: 21 marzo 2017 / Aceptado: 8 junio 2017 rado el de mayor patogenicidad e impacto económico en todo el mundo ${ }^{2,20}$.

La administración de drogas antiparasitarias constituye la principal alternativa para el control de los nematodes, pero el desarrollo creciente de la resistencia a los antihelmínticos está comprometiendo seriamente la sustentabilidad de esta tecnología. Por ello, la mayor 
preocupación actual es el aumento y la dispersión de aislamientos portando alelos con resistencia a múltiples antihelmínticos ${ }^{9}$.

En Argentina, aislamientos de Haemonchus contortus con resistencia múltiple a dos, tres y cuatro drogas con diferente modo de acción, han sido informados en ovinos de Corrientes y Entre Ríos ${ }^{16}$, así como en provincias del área central como Buenos Aires y Santa $\mathrm{Fe}^{5,18}$.

Al igual que en los ovinos, el género Haemonchus aparece como el de mayor prevalencia e importancia económica en los caprinos, al menos para el centro y noroeste de Argentina. En la región también son comunes los aislamientos con resistencia múltiple ${ }^{1,19}$. Esta situación involucra a nematodicidas de amplio espectro como levamisoles, bencimidazoles y lactonas macrocíclicas así como a drogas de espectro reducido como el closantel.

Actualmente las únicas drogas de uso en pequeños rumiantes con las que no ha sido documentada la resistencia antihelmíntica en nuestro país son el monepantel y el derquantel, introducidas al mercado veterinario argentino en el 2011 y 2015 respectivamente (Fiel C., comunicación personal), registradas para uso exclusivo en ovinos.

Sin embargo, en otros países de la región Mercosur como Uruguay y Brasil, la resistencia a la primera de estas drogas ya ha sido recientemente informada ${ }^{3,14}$. La única formulación conteniendo derquantel, se comercializa en todo el mundo en combinación con abamectina en un intento por mejorar su eficacia y demorar la aparición de resistencia antihelmíntica.

Los objetivos del presente trabajo fueron comunicar las observaciones de campo sobre la eficacia clínica de esta combinación, en el control de dos aislamientos de Haemonchus contortus con diferentes antecedentes de resistencia a lactonas macrocíclicas parasitando a una majada de ovinos y un hato caprino de las provincias de Santa Fe y Córdoba respectivamente.

\section{MATERIAL Y MÉTODOS}

Localización de las experiencias, antecedentes $y$ animales utilizados. Las experiencias se llevaron a cabo en el período comprendido entre agosto de 2016 y enero de 2017 en una majada de ovejas pampinta (Experiencia A) y un hato caprino (Experiencia B) pertenecientes a las estaciones experimentales del INTA de Rafaela y Deán Funes respectivamente. En la Experiencia A se evaluó la actividad de la combinación conteniendo $10 \mathrm{mg} / \mathrm{ml}$ de derquantel $+1 \mathrm{mg} / \mathrm{ml}$ de abamectina ("Startec", Zoetis-Pfizer Animal Health) sobre 10 ovejas (paridas entre 4 y 6 semanas antes del inicio de la experiencia) y dos grupos de 11 y 12 corderos (uno al pie de la madre y otro destetado, de aproximadamente dos y cuatro meses de edad respectivamente). La majada registraba antecedentes de resistencia a múltiples antihelmínticos de amplio y reducido espectro y en marzo de 2016 la eficacia de la moxidecti- na, la lactona de mayor actividad contra Haemonchus contortus, no superaba el 30\% (datos de INTA Rafaela, no publicados). En la experiencia B, se utilizaron 25 cabrillonas y cabras adultas de raza Anglo Nubian. El establecimiento registraba antecedentes de resistencia a ivermectina y febendazol. En evaluaciones realizadas en abril de 2016 la eficacia de la moxidectina fue de aproximadamente del 90\% (datos de INTA Rafaela, no publicados).

Diseño de las experiencias, tratamientos y determinaciones parasitológicas. La eficacia de la droga se determinó a través del test de reducción del conteo de huevos (TRCH) en heces. Entre los días -1 y 0 de cada una de las experiencias, los animales fueron pesados, identificados con caravanas y se tomaron muestras de materia fecal para determinar el número de huevos de nematodes por gramo de heces (hpg) mediante la técnica de McMaster modificada ${ }^{15}$. Para ambas experiencias se seleccionaron animales con valores de hpg mayores o iguales a 300. Alícuotas de materia fecal fueron obtenidas para la realización de coprocultivos y recuperación de larvas de tercer estadio a través de un aparato de Baermann, para identificación de los géneros de nematodes intervinientes. En ambas experiencias (A y B) todos los tratamientos con la combinación derquantel + abamectina se realizaron en el día 0 , en forma oral y a la dosis de $0,2 \mathrm{ml} / \mathrm{kg}$. Entre los días 13 y 15 post-tratamiento se tomaron nuevas muestras de materia fecal para establecer el TRCH.

Análisis de datos y criterios para la evaluación de susceptibilidad y/o resistencia. Para el TRCH se siguieron las recomendaciones generales de la World Association of Veterinary Parasitology ${ }^{4}$. En ambas experiencias se compararon los valores del hpg pre y post-tratamiento de los mismos animales utilizando la siguiente fórmula: TRCH $=100 \times$ (1-[T2/T1]), donde T2 es el promedio de los valores de hpg post-tratamiento y T1 el promedio de los valores de hpg pre-tratamiento ${ }^{13}$. En los corderos de la experiencia A, además de los valores promedios, el hpg de cada animal se utilizó para estimar la reducción individual de los mismos.

\section{RESULTADOS Y DISCUSIÓN}

Los coprocultivos pre-tratamiento de ovinos y caprinos mostraron la participación casi exclusiva del género Haemonchus con más del 98\% de las larvas clasificadas como pertenecientes a este género, mientras que con posterioridad a los tratamientos, tal porcentaje alcanzó el $100 \%$.

En la experiencia A (majada con antecedentes de marcada resistencia a lactonas macrocíclicas), el TRCH mostró una eficacia del 99,5\% en las ovejas tratadas durante el periparto, pero en las dos evaluaciones realizadas sobre los corderos, estos porcentajes fueron del 86,3 y del $77,6 \%$ respectivamente. Esta disimilitud en el TRCH entre las categorías de adultos y corderos po- 
dría ser el resultado de diferentes eficacias del derquantel sobre los distintos estadios de Haemonchus que estaban parasitando a los animales en el momento de los tratamientos.

La información de la acción del derquantel específicamente sobre Haemonchus contortus indica una actividad alta sobre estadios adultos, pero reducida sobre los estadios de larva de cuarto estadio o L $4{ }^{8}$. En el caso de las ovejas es conocido que el aumento de hpg en el periparto o alza de lactación, se produce en parte por la mayor oviposición de los nematodes adultos ya presentes en las mismas. En este contexto, la eficacia de la combinación antihelmíntica mayor al 99\% observada en las ovejas podría ser el resultado de la acción de la droga sobre estos estadios maduros que componen la mayor carga parasitaria en esta época del año.

Por el contrario, en los corderos, probablemente la proporción de L4 al momento del tratamiento constituía la mayor parte de la carga parasitaria y los menores porcentajes de reducción de los hpg podrían ser consecuencia de la mayor cantidad de estos estadios sobrevivientes al tratamiento con derquantel. En esta majada, con marcada resistencia a las lactonas, la actividad de la abamectina probablemente haya agregado poca (o ninguna) eficacia a la formulación evaluada.

Estudios de eficacia controlada (necropsias y recuento de nematodes) para la combinación derquantelabamectina, muestran un actividad alta sobre otros nematodes como Trichostrongylus colubriformes ${ }^{8}$ y Teladorsagia circumcincta ${ }^{6}$ con resistencia múltiple. En el caso específico de Haemonchus contortus sensibles a lactonas macrocíclicas como la ivermectina también han sido informadas eficacias mayores al $95 \%{ }^{11}$.

En nuestro país estudios de eficacia controlada llevados a cabo en 2015 muestran también una actividad mayor al 99\% en el control de aislamientos de Haemonchus contortus resistentes a los bencimidazoles (Fiel C., comunicación personal).

Por otra parte, los resultados del presente trabajo utilizando el TRCH, indican que en presencia de aislamientos de Haemonchus contortus con marcada resistencia a lactonas macrocíclicas, la inclusión de abamectina no mejora significativamente la actividad de la combinación. En estas situaciones, la actividad reducida del derquantel sobre L4 permitiría la sobrevida de algunos de estos estadios, produciendo porcentajes de eficacia inferiores a los considerados como deseables (mayores al 95\% para una formulación con un antihelmíntico único o mayores al $98 \%$ para combinaciones de antihelmínticos). Otros investigadores informaron que en corderos experimentalmente infectados con aislamientos de Haemonchus contortus con resistencia a la abamectina y tratados cuando la carga parasitaria estaba comprendida exclusivamente por L4, la actividad de esta combinación sobre dchos estadios resultó inferior al $20 \%$.

En el mismo sentido, nuestros hallazgos concuerdan con información proveniente de Australia (donde la resistencia a la abamectina es común), indicando que en establecimientos con resistencia a las lactonas, la eficacia de la combinación bajo estas particulares situaciones se encuentra reducida en porcentajes aproximados al 90\% ${ }^{12,17}$.

En la experiencia B (hato caprino con resistencia moderada a lactonas macrocíclicas) el TRCH mostró tanto en cabrillonas como cabras adultas, una eficacia superior al 99,5\% (Tabla 1). La combinación de derquantel + abamectina no está registrada para su uso en caprinos y en el presente trabajo la inclusión de esta especie se realizó exclusivamente con fines experimentales y las observaciones del presente estudio deberían ser evaluadas en este contexto.

La formación de la respuesta inmune, así como la expresión de la misma, es menos eficiente que en los ovinos, resultando en un mayor parasitismo en los caprinos ${ }^{7}$. Asímismo, en esta última especie la absorción de los antihelmínticos es menor que en otros rumiantes; también metabolizan y eliminan estas drogas en forma más rápida, por lo cual es recomendable una dosificación diferenciada (generalmente mayor) a la de los ovinos ${ }^{10}$.

En nuestra experiencia y al no existir antecedentes documentados sobre farmacocinética o toxicidad del derquantel en esta especie, se utilizó la dosis ovina. No obstante, la eficacia de la combinación en el hato experimental fue alta y similar a la observada en las ovejas adultas de la experiencia A. Los antecedentes en el hato caprino indicaban una moderada resistencia a las lactonas macrocíclicas y en esta particular situación, los efectos aditivos o incluso sinérgicos de la abamectina podrían haber contribuido al elevado porcentaje observado en el TRCH de esta experiencia.

En conclusión, este estudio indica que la nueva combinación de derquantel + abamectina puede resul- 
tar en diferentes eficacias para el control del género Haemonchus en función del status previo de susceptibilidad o resistencia a las lactonas macrocíclicas de cada población parasitaria. Estas observaciones demuestran una vez más, que bajo la situación generalizada de resistencia antihelmíntica en los nematodes de los pequeños rumiantes de nuestro país, no existe una opción química única (una "bala de plata"), por lo cual se enfatiza la necesidad de evaluar a nivel de cada establecimiento la actividad de todos los antiparasitarios, sean estos formulados con viejas o nuevas drogas. Los tratamientos combinados con antihelmínticos de distinto modo de acción son herramientas promisorias en el manejo de la resistencia, pero deberían ser integrados con el uso regular del TRCH para determinar bajo condiciones de campo la eficacia de estas prácticas de control.

Agradecimientos. Al convenio INTA-AUDEASCONADEV (CIAC 940143), al PE INTA (1115055) y a la Secretaría de Investigación de la Universidad Católica de Córdoba por la financiación del presente trabajo. Al Ing. Agr. Cristian Mohn, al Med. Vet. Alejandro Smulovitz y a Luciana Fassola y Franco Masnij por la colaboración en las tareas de campo y laboratorio.

\section{REFERENCIAS}

1. Aguirre D, Cafrune MM. 2013. Epidemiología e impacto productivo de nematodos en la región del NOA. En: Enfermedades parasitarias de importancia clínica y productiva en rumiantes, Ed. Hemisferio Sur, Montevideo, p.115-129.

2. Besier B. 2007. New anthelmintics for livestock: the time is right. Trends in Parasitol 23: 21-24.

3. Cintra MC, Teixeira VN, Nascimento LV, Sotomayor CS. 2016. Lack of efficacy of monepantel against Trichostrongylus colubriformis in sheep in Brazil. Vet Parasitol 216: 4-6.

4. Coles GC, Bauer C, Borgsteede FH, Geerts S, Klei TR, Taylor MA, Waller PJ. 1992. World Association for the Advancement of Veterinary Parasitology (WAAVP) methods for the detection of anthelmintic resistance in nematodes of veterinary importance. Vet Parasitol 44: 35-44.

5. Entrocasso C, Alvarez L, Manazza J, Lifcschitz A, Borda B, Virkel G, Mottier L, Lanusse C. 2008. Clinical efficacy assessment of the albendazole-ivermectin combination in lambs parasitized with resistant nematodes. Vet Parasitol 155: 249-256.

6. Geurden T, Hodge A, Winstanley D, Bartley D, Taylor M, Morgan C, Fraser S, Maeder S, Bartram D. 2012. The efficacy of a combined oral formulation of derquantelabamectin against anthelmintic resistant gastro-intestinal nematodes of sheep in the UK. Vet Parasitol 189: 308-316.

7. Hoste H, Sotirakis S, Landaus Y, Jackson F, Beveridge I. 2010. Goat-nematode interactions: think differently. Trends in Parasitol 26: 376-381.
8. Kaminsky R, Bapst B, Stein PA, Strehlau GA, Allan BA, Hosking BC, Rolfe PF, Sager H. 2011. Differences in efficacy of monepantel, derquantel and abamectin against multi-resistant nematodes of sheep. Parasitol Res 109: 19-23.

9. Kaplan RM. 2004. Drug resistance in nematodes of veterinary importance: a status report. Trends in Parasitol 20: 477-481.

10. Lespine A, Chartier C, Hoste H, Alvinerie M. 2012. Endectocides in goats: Pharmacology, efficacy and use conditions in the context of anthelmintics resistance. Small Rum Res 103: 10-17.

11. Little PR, Hodge A, Maeder SJ, Wirtherle NC, Nicholas DR, Cox GG, Conder GA. 2011. Efficacy of a combined oral formulation of derquantel-abamectin against the adult and larval stages of nematodes in sheep, including anthelmintic-resistant strains. Vet Parasitol 181: 180193.

12. Love S. 2016. Anthelmintics (drenches) for sheep, goats and alpacas. http://www. dpi.nsw.gov.au/animals-and-livestock/sheep/health/internal-parasites/registered-drenchessheep-worms.

13. McKenna PB. 2006. Further comparison of faecal egg count reduction test procedures: sensitivity and specificity. NZ Vet J 54: 365-366.

14. Mederos AE, Ramos Z, Banchero GE. 2014. First report of monepantel Haemon-chus contortus resistance on sheep farms in Uruguay. Parasit Vectors 7: 598.

15. Roberts F, O'Sullivan P. 1949. Methods for egg counts and larval culture for strongyles infesting gastrointestinal tract of cattle. Aust J Agric Res 1: 99-102.

16. Romero JR, Sánchez R, Boero C. 2007. Nematodes. Epidemiología y control. Epidemiología de la gastroenteritis verminosa de los ovinos en la pampa húmeda y la mesopotámica. En: Enfermedades parasitarias de los ovinos y otros rumiantes menores en el cono sur de América. INTA Publicación Técnica $\mathrm{N}^{\mathrm{o}}$ 70, p. 33-42.

17. Sales N, Love S. 2016. Resistance of Haemonhus spp to monepantel and reduced efficacy of a derquantel/abamectin combination confirmed in sheep in NSW. Vet Parasitol 228: 193-196.

18. Steffan P, Sánchez E, Entrocasso C, Fiel C, Lloberás M, Riva E, Guzmán M. 2011. Eficacia de monepantel contra nematodes de ovinos con resistencia antihelmíntica múltiple en la región templada de Argentina. Vet Arg 28: 1-12.

19. Suarez V, Fondraz M, Viñabal A, Martinez G, Salatin A. 2013. Epidemiología de los nematodes gastrointestinales en caprinos lecheros en los valles templados del NOA, Argentina. Rev RIA 39: 191-197.

20. Waller P. 2003. Global perspectives on nematode parasite control in ruminant livestock: the need to adopt alternatives to chemotherapy, with emphasis on biological control. Anim Health Res 4: 35-43. 